\title{
Corrections to "On the computation of the Nielsen numbers and the converse of the Lefschetz coincidence theorem"
}

(Fund. Math. 140 (1992), 191-196)

by

Peter Wong (Lewiston, Me.)

I thank Professor Boju Jiang for pointing out that Theorem 2.3 of [3] which is quoted as Lemma 1.2 of [1], is false in general. Consequently, without additional hypotheses, the main results in $[1, \S 2]$ do not hold in the generality as stated. Let $f, g: M_{1} \rightarrow M_{2}$ be as in [1,2.1]. In addition, we assume that $M_{1}, M_{2}$ are compact, $M_{1}$ is triangulable and $\pi_{1}\left(M_{1}\right)$ is finite so that the universal cover $\widetilde{M}_{1}$ is also compact. By a result of Schirmer, we may assume without loss of generality that the coincidence set of $f$ and $g$ is given by $C_{f, g}=\left\{x_{1}, \ldots, x_{k}\right\}$ such that each $x_{i}$ is a distinct coincidence class. It follows from $[1, \S 1]$ that each root class of $\eta: \widetilde{M}_{1} \rightarrow M_{2}$ must lie entirely inside the fiber $p_{1}^{-1}\left(x_{i}\right)$ over $x_{i}$ for some $i$. Following [2, Cor. 5], the root classes of $\eta$ have the same root index. Furthermore, $\eta$ has exactly $|K|=\left|\pi_{1}\left(M_{2}\right)\right|$ root classes if $\operatorname{deg} \eta \neq 0$. It is shown in the proof of [1, 2.1] that every point of $p_{1}^{-1}\left(x_{i}\right)$ has the same root index which coincides with the coincidence index at $x_{i}$. By summing all the indices, we obtain $\operatorname{deg} \eta=L(f, g) \cdot\left|\pi_{1}\left(M_{1}\right)\right|$.

Case (I): If $K$ is infinite, then $\operatorname{deg} \eta=0$ and hence every $x_{i}$ is inessential. Thus $N(f, g)=0$ and $f$ and $g$ are deformable to be coincidence free.

Case (II): Suppose that $K$ is finite. It follows that $\operatorname{deg} \eta=|K| \cdot \omega=$ $L(f, g) \cdot\left|\pi_{1}\left(M_{1}\right)\right|$ where $\omega$ is the root index of a root class of $\eta$. If $L(f, g)=0$ then $\operatorname{deg} \eta=0$ and hence $N(f, g)=0$. Again, $f$ and $g$ are deformable to be coincidence free. Let $r=\left|\pi_{1}\left(M_{2}\right)\right| /\left|\pi_{1}\left(M_{1}\right)\right|$. Now suppose that $L(f, g) \neq 0$.

1991 Mathematics Subject Classification: Primary 55M20.

Key words and phrases: fixed points, coincidences, roots, Lefschetz number, Nielsen number. 
If $\pi_{1}\left(M_{1}\right)=1$ then $M_{1}=\widetilde{M}_{1}$ and the $x_{i}$ 's have the same coincidence (root) index. Thus, $N(f, g)$ divides $L(f, g)$. If $\omega= \pm 1$, then every point in $p_{1}^{-1}\left(x_{i}\right)$ is a root class of index \pm 1 . Therefore, $N(f, g)=|L(f, g)|=r$. Moreover, if $\operatorname{gcd}\left(\omega,\left|\pi_{1}\left(M_{1}\right)\right|\right)=1$, it follows that every point in $p_{1}^{-1}\left(x_{i}\right)$ is a root class, which implies that the $x_{i}$ 's have the same coincidence index. Therefore, $N(f, g)$ divides $L(f, g)$.

We now summarize the above in the following

TheOREM A. Let $f, g: M_{1} \rightarrow M_{2}$ be maps between closed, connected, triangulable and orientable $n$-manifolds $(n \geq 3)$ such that $\left|\pi_{1}\left(M_{1}\right)\right|<\infty$ and $M_{2}=\widetilde{M}_{2} / K$ where $\widetilde{M}_{2}$ is a connected simply connected topological group and $K$ is a discrete subgroup. If $K$ is infinite or $L(f, g)=0$ then $N(f, g)=0$. Hence $f$ and $g$ are deformable to be coincidence free. If $K$ is finite and we let $r=|K| /\left|\pi_{1}\left(M_{1}\right)\right|, \omega=L(f, g) / r$ then

(1) $\pi_{1}\left(M_{1}\right)=1 \Rightarrow N(f, g)$ divides $L(f, g)$;

(2) $\omega= \pm 1 \Rightarrow N(f, g)=|L(f, g)|=r$;

(3) $\operatorname{gcd}\left(\omega,\left|\pi_{1}\left(M_{1}\right)\right|\right)=1 \Rightarrow N(f, g)$ divides $L(f, g)$ and $N(f, g)=r$.

Cor. 2.2 of [1] will hold true if $\left|\pi_{1}\left(M_{1}\right)\right|<\infty$. When $M_{1}=M_{2}, r=1$. Thus, for the fixed point case, we replace 2.3 and 2.4 of [1] by the following

Corollary B. Let $M_{1}, M_{2}$ be as in Theorem A and $M_{1}=M_{2}=M$. Let $f: M \rightarrow M$ be a map. If $L(f)=0$ then $N(f)=0$ and $f$ is deformable to be fixed point free. If (i) $L(f)= \pm 1$ or (ii) $\operatorname{gcd}\left(L(f),\left|\pi_{1}\left(M_{1}\right)\right|\right)=1$, then $N(f)=1$.

It is worthwhile to note that if $M_{1}$ is compact and $M_{2}$ is a compact Lie group, then it can be shown easily, along the lines of [1], that the coincidence classes of $f$ and $g$ are the root classes of $\varphi$, where $f, g: M_{1} \rightarrow M_{2}$ and $\varphi: M_{1} \rightarrow M_{2}$ is given by $\varphi(x)=f(x)^{-1} g(x)$. Furthermore, the coincidence index of $f$ and $g$ is the same as the root index of $\varphi$. Hence we have the following

TheOREM C. Let $f, g: M_{1} \rightarrow M_{2}$ be maps from a closed connected oriented $n$-manifold $M_{1}(n \geq 1)$ to a compact connected Lie group $M_{2}$ of the same dimension. If the Lefschetz coincidence number $L(f, g)=0$ then the Nielsen coincidence number $N(f, g)=0$. Otherwise, $N(f, g)>0$ and $N(f, g)$ divides $L(f, g)$.

I thank the referee for some helpful suggestions and comments.

\section{References}

[1] P. Wong, On the computation of the Nielsen numbers and the converse of the Lefschetz coincidence theorem, Fund. Math. 140 (1992), 191-196. 
[2] R. Brooks, Certain subgroups of the fundamental group and the number of roots of $f(x)=a$, Amer. J. Math. 95 (1973), 720-728.

[3] R. Brooks and P. Wong, On changing fixed points and coincidences to roots, Proc. Amer. Math. Soc. 115 (1992), 527-533.

DEPARTMENT OF MATHEMATICS

BATES COLLEGE

LEWISTON, MAINE 04240

U.S.A.

Received 24 April 1992;

in revised form 22 May 1992 